\title{
HIV-specific cytolytic CD4 T-cell responses effectively control HIV infection in macrophages
}

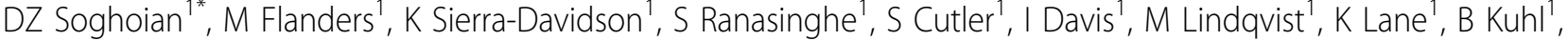 \\ G Kranias ${ }^{1}$, A Piechocka-Trocha' ${ }^{1}$, H Jessen ${ }^{2}$, BD Walker ${ }^{1}$, H Streeck ${ }^{1}$
}

From AIDS Vaccine 2012

Boston, MA, USA. 9-12 September 2012

\section{Background}

HIV-specific cytolytic CD4 T-cell responses expand during acute HIV infection in individuals who control viremia and are associated with better disease outcome. Up to $75 \%$ of the HIV-specific CD4 T-cells exhibit a cytolytic phenotype during acute infection, but it is not understood how cytolytic CD4 T-cells contribute to viral control or what their primary target cells are.

\section{Methods}

Using a novel, fluorescence-based single-round viral suppression assay, we assessed the ability of CD4 T-cells from HIV infected subjects to lyse infected macrophages. Elimination of infected macrophages and CD4 cytolytic phenotype were determined by flow cytometry. In addition, HIV-specific CD4 T-cell clones were generated and their cytolytic ability examined by Cr51-release and viral inhibition assays.

\section{Results}

We observed significantly higher degranulatory HIV-specific CD4 T-cell responses in HIV controllers compared to progressors $(\mathrm{p}=0.015)$. Moreover, about $1 / 4$ of all HIV-specific CD4 T-cell clones showed cytolytic activity by $\mathrm{Cr}$ 51-release. Using a single-round viral suppression assay, we additionally observed that HIV-specific CD4 $\mathrm{T}$-cells from chronically infected subjects were able to significantly lyse HIV infected macrophages $(p=0.004)$. Elimination of HIV-infected macrophages was dosedependent, up to $37 \%$ at $\mathrm{E}: \mathrm{T}=5: 1$. Lytic ability could be observed ex vivo, and was enhanced after short term Gag-specific expansion in culture ( $11 \%$ to $32 \%, \mathrm{p}=0.014)$. Furthermore, we observed that the inhibitory capacity of
CD4 $\mathrm{T}$ cells could be abrogated using an HLA-DR blocking antibody. CD4 T-cell-mediated macrophage lysis was associated with strong HIV-specific cytolytic activity by intracellular cytokine staining and high expression of granzymes/perforin within HIV-specific CD4 T-cells.

\section{Conclusion}

Our data demonstrate that HIV-specific CD4 T-cells derived from infected individuals have the ability to eliminate infected macrophages. These data suggest a role for HIV-specific cytolytic CD4 T-cell responses, in the absence of CD8 $\mathrm{T}$ cell responses, in the lysis of HIV-infected macrophages, which represent important reservoirs for viral infection and viral dissemination.

\section{Author details}

'Ragon Institute of MGH, MIT, and Harvard, Charlestown, MA, USA. ${ }^{2}$ Praxis Jessen Jessen Stein, Berlin, Germany.

Published: 13 September 2012

doi:10.1186/1742-4690-9-S2-P274

Cite this article as: Soghoian et al: HIV-specific cytolytic CD4 T-cell responses effectively control HIV infection in macrophages. Retrovirology 2012 9(Suppl 2):P274.

${ }^{1}$ Ragon Institute of MGH, MIT, and Harvard, Charlestown, MA, USA

Full list of author information is available at the end of the article 